\title{
Choriocarcinoma of unknown origin with multiple organ metastasis and cerebral hemorrhage: A case report and literature review
}

\author{
HONGTAO WEI $^{1 *}$, TIANPENG ZHANG $^{1 *}$, BING LIU $^{1}$, XIAOWEI XUE ${ }^{2}$ and GUOXING WANG ${ }^{1}$ \\ ${ }^{1}$ Department of Emergency, Beijing Friendship Hospital, Capital Medical University, Beijing 100050; \\ ${ }^{2}$ Department of Pathology, Peking Union Medical College Hospital, Chinese Academy of Medical Sciences, \\ Beijing 100730, P.R. China
}

Received March 11, 2015; Accepted March 18, 2016

DOI: $10.3892 / \mathrm{ol} .2016 .4463$

\begin{abstract}
A 26-year-old man was admitted to Beijing Friendship Hospital, Capital Medical University (Beijing, China) with a 4-day history of headache, moderate fever and numbness in the right upper limb. Prior to this, the patient had been diagnosed with cerebral hemorrhage by computed tomography (CT) scan upon visiting a local hospital. Chest $\mathrm{X}$-ray revealed multiple lesions in the lungs. Following referral, no abnormalities were found elsewhere, including in the testes, during a physical examination. Additional examination of other tumor biomarkers was unremarkable, and the initial suspicion of parasitic infection was ruled out. Tests revealed extremely high levels of $\beta$-human chorionic gonadotropin (>200,000 mIU/ml). In addition, CT scans showed multiple metastases in the head, lungs, liver and kidneys. An ultrasound-guided Tru-Cut biopsy of the liver was performed in order to form a definitive diagnosis. Although the patient was treated with mannitol to reduce intracranial pressure, and with cefoperazone sodium and sulbactam sodium to fight infection, the patient succumbed to a cerebral hernia on the fourth day of hospitalization. Following this, the ultrasound-guided Tru-Cut liver biopsy result was received, which suggested a diagnosis of choriocarcinoma.
\end{abstract}

\section{Introduction}

Choriocarcinoma of non-placental origin is highly malignant and is mainly characterized by the production of large amounts

Correspondence to: Mr. Guoxing Wang, Department of Emergency, Beijing Friendship Hospital, Capital Medical University, 95 Yong-an Road, Xi-Cheng, Beijing 100050, P.R. China

E-mail:wgx_zn@aliyun.com

*Contributed equally

Key words: choriocarcinoma, human chorionic gonadotropin, malignant carcinoma, male, liver biopsy of human chorionic gonadotropin (HCG) hormones (1). The disease is extremely rare, particularly in male patients, and there are few studies on such cases (2). The present study reports the case of a 26-year-old male patient with choriocarcinoma, providing a firsthand opportunity to review the specific features of the disease, including rapid and multiple metastasis, a high level of $\beta-\mathrm{HCG}$, and the associated life-threatening effects.

\section{Case report}

In March 2013, a 26-year-old male with a 4-day history of headache, moderate fever and numbness in the right upper limb was diagnosed with a cerebral hemorrhage by computed tomography (CT) scan at The General Hospital of the Second Artillery Corps of Chinese PLA (Beijing, China). On the same day, the patient was transferred to Beijing Friendship Hospital, Capital Medical University (Beijing, China) due to the identification of multiple undefined lesions in the lungs by X-ray. The patient did not have any chronic illness and lived in a house with two pets, a python and a dog, suggesting the possibility of a parasitic disease.

Upon examination, it was observed that the patient had normal vital signs with a Glasgow coma scale of 14 . No palpable enlargement of the lymph nodes or lumps beneath the skin were observed, however, superficial sensation was slightly impaired. Muscle strength of the right upper limb was measured at level 4, according to manual muscle testing (3), and the right Babinski sign was positive. The patient's testes were also examined, but no abnormalities were found.

Chest X-ray and chest CT scans showed multiple lesions in the lungs (Fig. 1). Furthermore, CT scans also showed multiple tumors in the head, liver and kidneys (Figs. 2 and 3). The laboratory results were as follows: White blood cell count, $16.59 \times 10^{9} / 1$ (normal, 4-10x10 $/ 1$ ); hemoglobin, $131 \mathrm{~g} / 1$ (normal, 120-160 g/l); platelets, $277 \times 10^{9} / 1$ (normal, $100-300 \times 10^{9} / 1$ ); C-reactive protein, $132 \mathrm{mg} / \mathrm{l}$ (normal, $0-8 \mathrm{mg} / \mathrm{l}$ ); alanine aminotransferase, $93 \mathrm{U} / 1$ (normal, 0-40 U/1); total bilirubin, $25.6 \mu \mathrm{mol} / 1$ (normal, 3.42-17.1 $\mu \mathrm{mol} / \mathrm{l}$ ); direct bilirubin, $16.62 \mu \mathrm{mol} / 1$ (normal, $0-6.84 \mu \mathrm{mol} / \mathrm{l}$ ) and albumin, $25.7 \mathrm{~g} / \mathrm{l}$ (normal, 35-55 g/1). Parameters for blood electrolytes and 

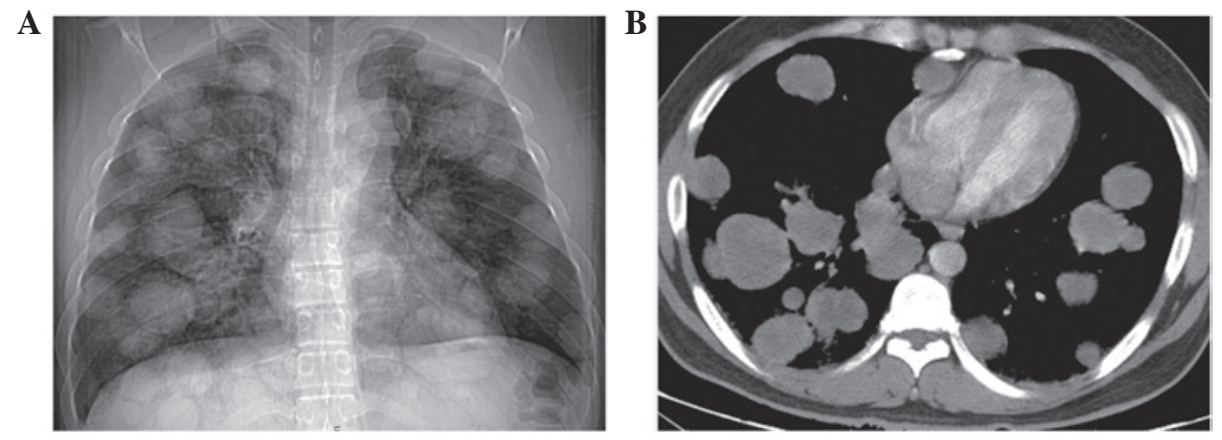

Figure 1. X-ray and contrast-enhanced computed tomography (CT) scan of the chest documenting diffuse metastatic cancer. (A) Chest X-ray with multiple lesions in the lungs. (B) Chest CT scan showing bilateral pulmonary nodules.
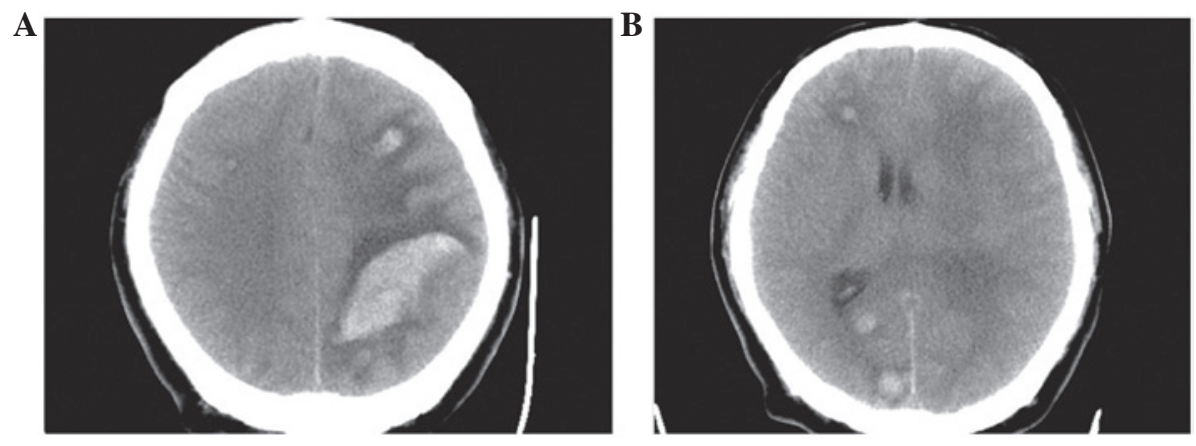

Figure 2. Brain computed tomography scans suggestive of intracranial hemorrhage and intracranial metastatic tumors. (A) Cerebroventricular haemorrhage and midline shift of the brain. (B) Multiple metastatic tumors.
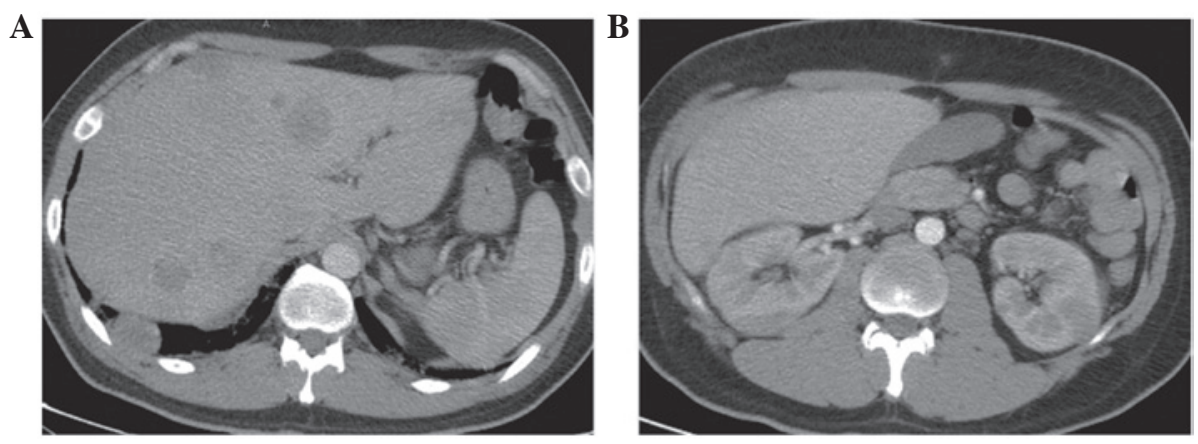

Figure 3. Multiple metastases of the liver and kidneys. Computed tomography scans showing (A) multiple low density lesions in the liver and (B) metastatic tumors in the kidneys.

renal function were within normal limits. For the differential diagnosis, tests were performed for angiotensin-converting enzyme (ACE), a few parasite specific antibodies (echinococcosis antibody, trichinosis antibody, schistosoma antibody and liver fluke antibody) and serum tumor markers. The presence of ACE within the normal range ruled out sarcoidosis. A negative result for the specific antibodies for echinococcosis, trichinosis, schistosoma and liver flukes ruled out the possibility of parasitic diseases. Moreover, the cancer biomarkers of $\alpha$-fetoprotein, carbohydrate antigen (CA)125 and CA199 were detected at normal levels. However, the levels of carcinoembryonic antigen, cytokeratin fragment 21-1 (CYF211) and neuron-specific enolase (NSE) were higher than normal (Table I). Most noticeably, the $\beta$-HCG level was much higher than normal (>200,000 mIU/ml; normal, 0-25 mIU/ml), as shown in Table I. This reinforced the suspicion of a malignant tumor. An ultrasound-guided Tru-Cut biopsy of the liver was performed to confirm this diagnosis.

In the meantime, the neurosurgeon suggested a conservative treatment, as it was believed that the prognosis could not be improved by surgery. Mannitol $(0.5 \mathrm{~g}$ by intravenous drip every $8 \mathrm{~h}$ ) was administered to the patient to reduce intracranial pressure. In addition, due to pyrexia and a high white blood cell count, cefoperazone sodium and sulbactam sodium were administered by injection ( $3.0 \mathrm{~g}$ by intravenous drip every $12 \mathrm{~h}$ ) to empirically fight infection. However, the patient's physical condition continued to deteriorate. After the third day of hospitalization, the patient developed a brain hernia, and finally, on the fourth day, the patient succumbed. Following this, the ultrasound-guided Tru-Cut liver biopsy 
Table I. Patient's serum tumor markers.

\begin{tabular}{lcc}
\hline Component & Value & Reference range \\
\hline $\mathrm{CEA}, \mathrm{ng} / \mathrm{ml}$ & 3.42 & $0.00-5.00$ \\
$\mathrm{AFP}, \mathrm{ng} / \mathrm{ml}$ & 4.11 & $0.00-15.00$ \\
$\mathrm{CA} 199, \mathrm{U} / \mathrm{ml}$ & 27.99 & $0.00-37.00$ \\
$\mathrm{CYF} 211^{\mathrm{a}}, \mathrm{ng} / \mathrm{ml}$ & 29.00 & $0.10-3.30$ \\
$\mathrm{NSE}^{\mathrm{a}}, \mathrm{ng} / \mathrm{ml}$ & 39.36 & $0.00-16.30$ \\
$\mathrm{CA} 125^{\mathrm{a}}, \mathrm{U} / \mathrm{ml}$ & 76.60 & $0.00-35.00$ \\
$\beta-\mathrm{HCG}^{\mathrm{b}}, \mathrm{mIU} / \mathrm{ml}$ & $>200,000$ & $0-25$
\end{tabular}

${ }^{a}$ Value is higher than the reference range; ${ }^{b}$ value is much higher than the reference range. CEA, carcinoembryonic antigen; AFP, $\alpha$-fetoprotein; CA199, carbohydrate antigen 19-9; CYF211, cytokeratin fragment 21-1; NSE, neuron-specific enolase; CA125, carbohydrate antigen 125 ; $\beta$-HCG, $\beta$-human chorionic gonadotropin.

result was received and it was established that the carcinoma originated from the epithelial tissue (Fig. 4), thus supporting the diagnosis of choriocarcinoma.

\section{Discussion}

It is known that the majority of choriocarcinomas are placental choriocarcinomas, which always occur in females following gestational events such as molar pregnancy, normal or ectopic pregnancy, and abortion (1). Choriocarcinoma from non-placental origin is a rare disease, especially in male patients (4). A search of PubMed using keywords such as 'choriocarcinoma, non-gestational' and 'male', revealed only $\sim 20$ studies, and almost all of these were case reports. This further emphasizes the rareness of the carcinoma.

The 26-year-old patient in the present study was diagnosed with intracranial hemorrhage as the first symptom. Multiple lesions were then found in the lungs, liver and kidneys. Notably, no abnormalities were observed in the testicular physical exam. Choriocarcinoma usually undergoes vascular invasion and easily causes metastatic bleeding (4,5). A study by Baagar et al described choriocarcinoma syndrome as the hemorrhage of the tumor at its metastatic site. A case was reported in which a patient succumbed from the hemorrhage of a liver metastasis (5). There is no effective therapeutic treatment for choriocarcinoma syndrome, as it has already metastasized by the time it is diagnosed, and it is too late for the patient to undergo surgery.

In the present case, lesions were identified in the brain, lungs, liver, kidneys and skin. The presence of multiple lesions in the lung was particularly unique. It is extremely difficult to determine the location of the original carcinoma in patients where multiple organs are involved. When the present patient was first admitted, parasitic infections were tested for first due to a close proximity with dogs and snakes, and as the patient was also an animal breeder. Therefore, parasitic infections such as echinococcosis, trichinosis, schistosoma and liver flukes were first ruled out. In addition, the patient's ACE levels were also determined to rule out sarcoidosis. Furthermore, the possibility of metastatic carcinoma, particularly those
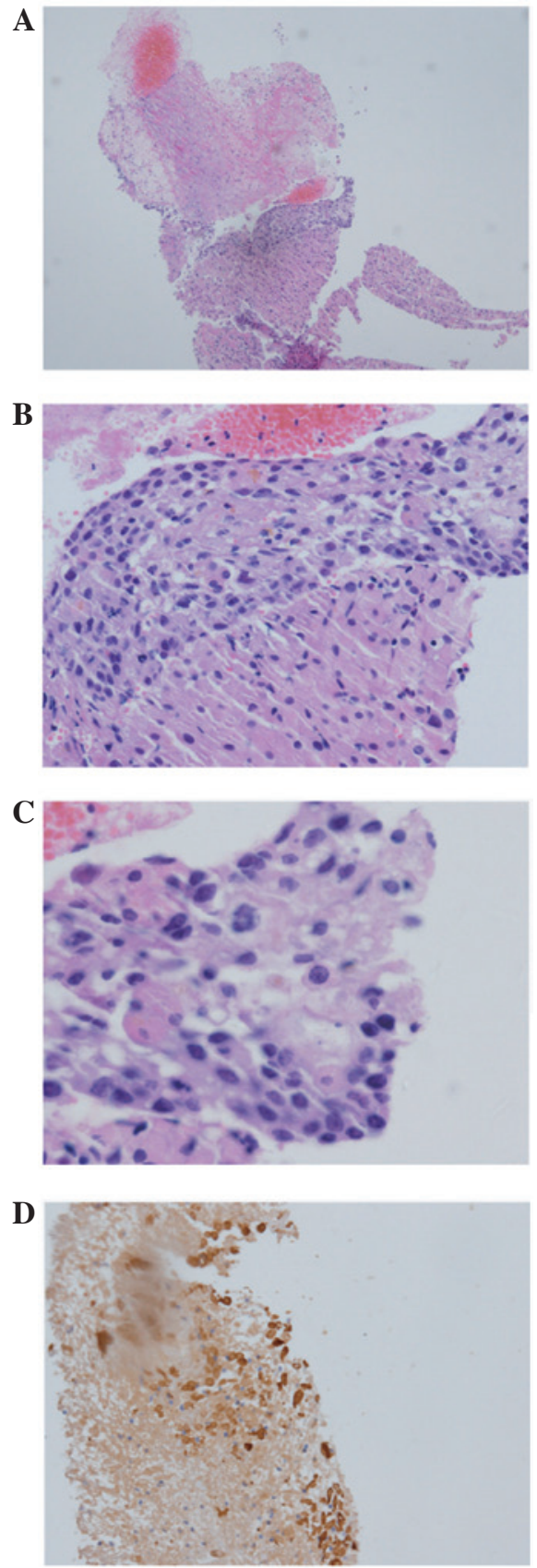

Figure 4. Histopathological characteristics of the hepatic metastasis biopsy. (A) Tumor cells surrounded by normal liver cells in the liver biopsy specimen (hematoxylin and eosin staining; magnification, x100). (B) Cells with severe heterotypical change corresponding to metastases (hematoxylin and eosin staining; magnification, x200). (C) Several mitoses are apparent in the specimen (hematoxylin and eosin staining; magnification, x400). (D) Metastatic tumor cells indicating a positive reaction for cytokeratin (immunohistochemical staining for cytokeratin; magnification, x100).

originating from the reproductive system, was considered. However, testicular examination did not find any abnormalities. Finally, after detecting high levels of $\beta-\mathrm{HCG}$, the possibility of a rare carcinoma was considered. Later, a liver biopsy provided further evidence that confirmed this diagnosis.

The HCG hormone is a characteristic marker of choriocarcinoma. A literature review by Yokoi et al reported that $96.4 \%$ of choriocarcinoma patients had abnormal elevated serum $\beta$-HCG levels (4). Jiang et al suggested that serum $\beta$-HCG levels could be used as a marker to assess the effectiveness 
of treatment (6). Since choriocarcinoma cells express high levels of HCG, we hypothesize that HCG levels may have a certain association with prognosis. A higher HCG level would correspond with a poor prognosis, but this hypothesis would require large samples to test it further. Other tumor markers in the present patient, including CA125, CYF211 and NSE, were exhibited at elevated levels, but the causes and significance of these increased levels are unknown.

It has also been reported that choriocarcinoma patients could develop paraneoplastic syndromes such as hyperthyroidism $(4,7,8)$. However, in the present case, thyroid function could not be checked due to a delayed diagnosis and the rapid deterioration of the patient's condition.

There have been no reports that describe a perfect treatment plan for choriocarcinoma of non-placental origin. Early tumor resection is believed to be an effective method (9). However, the best time for surgery is always missed when carcinoma is identified due to the high degree of malignancy and early bleeding. Thereafter, the only option left is conservative treatment such as chemotherapy, which includes treatment with drugs such as etoposide, cisplatinum, methotrexate, actinomycin D, bleomycin, 5-fluorouracil, VP-16, cyclophosphamide and vincristine (10-12). However, these drugs are only partially effective, as the majority of patients succumb within a short follow-up time. Guo et al reported one case of intracranial choriocarcinoma that was treated using radiotherapy, in which patient survived for $\sim 6$ months (13). However, this was only an individual case, and there has been no evidence of a large number of cases showing the effectiveness of conservative treatment. Only symptomatic treatments, such as hydration and anti-infection treatments, were administered to the present patient due to delayed diagnosis, however, we suggest that a patient should be given chemotherapeutic treatment as soon as a malignant tumor is suspected.

Choriocarcinoma of non-placental origin is a highly malignant carcinoma with poor prognosis. The present patient serves as a typical example to this effect, since multi-organ metastasis was observed, the symptoms were only displayed for 4 days prior to admission and the patient succumbed after the fourth day of admission. Similarly, based on literature reviews, the majority of patients succumb to organ bleeding, respiratory failure, hemorrhagic shock, disseminated intravascular coagulation and abdominal compartment syndrome (11). The occurrence of liver or brain metastases is associated with a worse prognosis (14).

In conclusion, the typical symptoms of multiple space-occupying lesions in different organs and accompanying high $\mathrm{HCG}$ levels point towards the possible diagnosis of choriocarcinoma of non-placental origin. This disease is life-threatening and should be diagnosed and treated as soon as possible.

\section{Acknowledgements}

This study was sponsored by the National Natural Science Funds of China (grant no. 813740041020795).

\section{References}

1. Smith HO, Kohorn E and Cole LA: Choriocarcinoma and gestational trophoblastic disease. Obstet Gynecol Clin North Am 32: 661-684, 2005.

2. Kyriakou F, Vaslamatzis MM, Bastani S, Lianou MA, Vourlakou C and Koutsoukou A: Primary choriocarcinoma of the renal pelvis presenting as intracerebral hemorrhage: A case report and review of the literature. J Med Case Rep 5: 501, 2011.

3. Hislop H, Avers D and Brown M (eds): Daniels and Worthingham's Muscle Testing: Techniques of Manual Examination and Performance Testing. 9th edition. Elsevier, India, 2013.

4. Yokoi K, Tanaka N, Furukawa K, Ishikawa N, Seya T, Horiba K, Kanazawa Y, Yamada T, Ohaki Y and Tajiri T: Male choriocarcinoma with metastasis to the jejunum: A case report and review of the literature. J Nippon Med Sch 75: 116-121, 2008.

5. Baagar K, Khan FY and Alkuwari E: Choriocarcinoma syndrome: A case report and a literature review. Case Rep Oncol Med 2013: 697251, 2013.

6. Jiang L, Wu JT and Peng X: Primary choriocarcinoma of the colon: A case report and review of the literature. World J Surg Oncol 11: 23, 2013.

7. Heda $P$ and Cushing G: Testicular choriocarcinoma presenting as hyperthyroidism. Am J Med 126: e1-e2, 2013.

8. McCracken EJ, Johnston PC, Lindsay JR, Mulholland C, McAleer JJ and Black RN: Testicular choriocarcinoma: An unusual case of paraneoplastic thyrotoxicosis. QJM 105: 675-677, 2012.

9. Bolze PA, Attia J, Massardier J, Seckl MJ, Massuger L, van Trommel N, Niemann I, Hajri T, Schott AM and Golfier F; EOTTD group: Formalised consensus of the European Organisation for Treatment of Trophoblastic Diseases on management of gestational trophoblastic diseases. Eur J Cancer 51: 1725-1731, 2015.

10. Waseda Y, Komai Y, Yano A, Fujii Y, Noguchi N and Kihara K: Pathological complete response and two-year disease-free survival in a primary gastric choriocarcinoma patient with advanced liver metastases treated with germ cell tumor-based chemotherapy: A case report. Jpn J Clin Oncol 42: 1197-1201, 2012.

11. Hadgu A, Tindni A and Panda M: Primary pulmonary choriocarcinoma in a male. BMJ Case Rep 2010: bcr0220102712, 2010.

12. Oshima J,Uemura M, Kato T, Nagahara A, Kiuchi H, Tsujimura A and Nonomura N: Modified BEP regimen leads to abatement of choriocarcinoma syndrome in a patient with extra gonadal germ cell tumor: A case report. Hinyokika Kiyo 60: 183-187, 2014 (In Japanese).

13. Guo J, Zhong C, Liu Q, Xu J, Zheng Y, Xu S, Gao Y, Guo Y, Wang Y, Luo Q and Jiang J: Intracranial choriocarcinoma occurrence in males: Two cases and a review of the literature. Oncol Lett 6: 1329-1332, 2013.

14. Chapman GW Jr: Metastatic intracerebral choriocarcinoma in a teenager. J Natl Med Assoc 89: 758-760, 1997. 